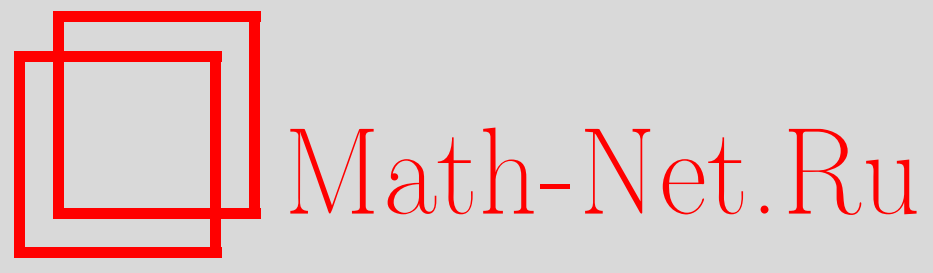

Е. А. Коровайцева, Применение метода дифференцирования по параметру в решении нелинейных задач стационарной динамики осесимметричных мягких оболочек, Вестн. Сам. гос. техн. унта. Сер. Физ.-мат. науки, 2021, номер 3, 556-570

DOI: https://doi.org/10.14498/vsgtu1855

Использование Общероссийского математического портала MathNet.Ru подразумевает, что вы прочитали и согласны с пользовательским соглашением

http://www.mathnet.ru/rus/agreement

Параметры загрузки:

IP: 52.90 .164 .192

26 апреля 2023 г., 16:51:20

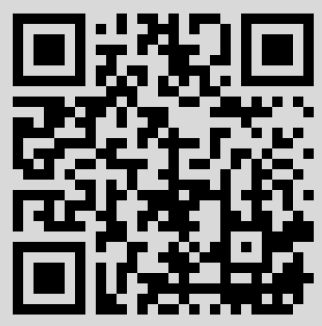


УДК 539.3

\title{
Применение метода дифференцирования по параметру в решении нелинейных задач стационарной динамики осесимметричных мягких оболочек
}

\author{
(C) E. А. Коровайщева
}

Московский государственный университет имени М. В. Ломоносова,
Научно-исследовательский институт механики,
Россия, 119192, Москва, Мичуринский проспект, 1.

\begin{abstract}
Аннотация
Предложен алгоритм решения задач о нелинейном динамическом поведении осесимметричных неразветвленных мягкооболочечных конструкций, основанный на использовании метода дифференцирования по параметру. Алгоритм не накладывает каких-либо ограничений на диапазон деформаций и перемещений, свойства материала, условия закрепления или форму меридиана конструкции. При этом уравнения движения в частных производных сводятся к нелинейным обыкновенным дифференциальным уравнениям с использованием метода прямых. Полученная система уравнений дифференцируется по календарному параметру. В результате решение задачи сводится к решению двух взаимосвязанных задач - квазилинейной многоточечной краевой задачи и нелинейной задачи Коши с правой частью специального вида. Особенности использования данного алгоритма применительно к задачам динамики мягких оболочек проявляются при его программной реализации и описаны в работе. Тестирование алгоритма выполнено на примере решения задачи динамического раздувания шарнирно опертой полусферы из неогуковского материала. Отмечено, что хотя формально рассматриваемая в примере оболочка не является составной, для построения численного решения необходимо использование метода сегментации интервала интегрирования по координате, что соответствует анализу составной конструкции. Исследовано влияние выбора шага по времени и схемы аппроксимации ускорения на результаты решения.
\end{abstract}

Ключевые слова: мягкая оболочка, высокоэластичный материал, динамическое раздувание, метод прямых, метод дифференцирования по параметру, физическая нелинейность, геометрическая нелинейность.

Получение: 23 марта 2021 г. / Исправление: 17 мая 2021 г. /

Принятие: 25 августа 2021 г. / Публикация онлайн: 30 сентября 2021 г.

\section{Научная статья}

○ (ㄱ) Контент публикуется на условиях лицензии Creative Commons Attribution 4.0 International (https://creativecommons.org/licenses/by/4.0/deed.ru)

\section{Образец для цитирования}

Ко ровайцева Е. А. Применение метода дифференцирования по параметру в решении нелинейных задач стационарной динамики осесимметричных мягких оболочек // Вестн. Сам. гос. техн. ун-та. Сер. Физ.-мат. науки, 2021. Т. 25, № 3. С. 556-570. https://doi.org/10.14498/vsgtu1855.

\section{Сведения об авторе}

Екатерина Анатольевна Коровайцева (10 https://orcid.org/0000-0001-6663-8689 кандидат технических наук; старший научный сотрудник; лаб. динамических испытаний; e-mail: katrell@mail.ru 
Введение. Задачи динамического деформирования мягких оболочек в доступной литературе освещены лишь в ограниченном количестве публикаций, а работы зарубежных авторов, по сути, касаются лишь трех частных простейших задач. Очевидно, причиной этого является вычислительная сложность рассматриваемой проблемы. Уравнения динамического деформирования мягких оболочек как систем с распределенной массой сформулированы в работах [1-5]. При этом в [1] рассматриваются лишь стационарные колебания оболочек конкретных форм и условий закрепления, а решения построены в аналитической форме. В работах [2,3] постановки и решения задач даны для оболочек специфической формы - каркасированных абсолютно гибкими дискретно расположенными нитями. Во всех упомянутых работах, несмотря на то, что постановка задачи дается для материала с произвольными нелинейными физическими соотношениями, результаты расчета приводятся лишь для линейного поведения материала оболочек (за исключением тестового примера удара по гибкой нити - квадратичной зависимости усилия от деформации нити). Авторами $[2,3,5]$ используются метод конечных разностей и метод конечных элементов. Примечательно, что все расчеты нестационарного динамического поведения мягкооболочечных конструкций указанных авторов являются решениями конкретных прикладных задач парашютостроения. Таким образом, применимость или особенности применения данных методов для случая истинной физической нелинейности материала и истинно больших деформаций остались неизученными.

В зарубежной литературе, за исключением монографии [4], не уделяется внимания построению разрешающих соотношений динамического деформирования мягкооболочечных конструкций в общей математической постановке. Однако множество работ посвящено решению соответствующих частных задач. Принципиальным отличием в подходе к постановке задач динамики от работ отечественных исследователей является отсутствие прикладной направленности задачи. Начиная с первых работ 60-х годов XX века до работ 2020 года зарубежными авторами рассматриваются лишь задачи динамического раздувания цилиндрической и сферической оболочек и плоских мембран [6-16]. Исходные данные задач отличаются формами упругих потенциалов материала, толщиной стенки рассматриваемой оболочки, зависимостью нагрузки от времени. Разрешающие соотношения во всех работах сводятся к уравнению движения системы с одной степенью свободы. В абсолютном большинстве работ получены аналитические решения этого уравнения и исследуются свойства диаграмм нагружения и фазовых кривых. Численное исследование сводится как максимум к решению системы обыкновенных дифференциальных уравнений методом Рунге-Кутта [17], методом конечных разностей [18], методом множественной пристрелки с использованием встроенной процедуры языка программирования ФОРТРАН [19] и методом конечных элементов в комплексе ABAQUS [20,21]. Подчеркнем, что каждый раз система разрешающих уравнений формулируется для конкретной рассматриваемой задачи, т.е. речь о разработке универсального алгоритма решения задач динамики мягкооболочечных конструкций не идет.

Можно выделить лишь две более сложные постановки задачи, рассмотренные зарубежными исследователями. Одна из них посвящена анализу прыжка мягкого актюатора, надуваемого жидкостью [22]. Однако в указан- 
ной работе решение задачи динамики сводится к решению системы обыкновенных дифференциальных уравнений для системы с двумя степенями свободы, причем для построения уравнений используются результаты квазистатического решения, полученного с использованием конечноэлементного комплекса ABAQUS, а далее применяется встроенная процедура MATLAB решения систем обыкновенных дифференциальных уравнений ODE45. В задачах второго типа $[23,24]$ исследуется распространение нестационарных волн в гиперупругом теле. Решения соответствующих систем ищутся в аналитическом виде.

Подводя итог обзора, можно заключить, что несмотря на проводимые исследования задач динамики мягкооболочечных конструкций на протяжении более 60-ти лет, до сих пор не существует ни их математической постановки, ни вычислительного алгоритма, одновременно ориентированных на решение задач максимально широкого класса и удобных для использования в численной реализации. Большие перемещения и деформации в задачах динамики численными методами не получены, а сопутствующие этому вычислительные трудности лишь декларируются отдельными исследователями, но до сих пор не изучены.

В настоящей работе предлагается математическая постановка и алгоритм решения задачи осесимметричного динамического деформирования составной мягкооболочечной конструкции как системы с распределенной массой и исследуются особенности реализации алгоритма на примере тестовой задачи о динамическом раздувании полусферы из неогуковского материала.

1. Математическая постановка и алгоритм решения. Пусть задача динамического деформирования мягкой оболочки описывается системой дифференциальных уравнений в векторно-матричной форме

$$
\frac{\partial \vec{y}_{i}}{\partial x_{i}}=\vec{f}_{i}\left(x_{i}, \vec{y}_{i}, \vec{z}_{i}, \vec{\mu}_{i}, \vec{q}_{i}\right)+M_{i} \frac{\partial^{2} \vec{y}_{i}}{\partial t^{2}}, \quad i \in[1, N]
$$

и системой дополнительных алгебраических соотношений

$$
\vec{\varphi}_{i}\left(x_{i}, \vec{y}_{i}, \vec{z}_{i}, \vec{\mu}_{i}, \vec{q}_{i}\right)=\overrightarrow{0}
$$

Соотношения $(1),(2)$ дополняются начальными условиями

$$
\vec{y}(x, 0)=\vec{y}_{0}, \quad \vec{y}^{\prime}(x, 0)=\vec{y}_{0}^{\prime},
$$

условиями сопряжения сегментов конструкции

$$
\vec{y}_{j}\left(x_{j, e}\right)=\vec{y}_{j+1}\left(x_{j+1, b}\right)+\vec{d}_{j+1} ; \quad j \in[1, N-1]
$$

и граничными условиями

$$
\vec{\psi}_{1}\left(x_{1}, \vec{y}_{1}, \vec{q}, \vec{\mu}, t\right)=\overrightarrow{0}, \quad 1 \rightleftharpoons 2
$$

Здесь $\quad \vec{y}_{i}$ - вектор-функция $n$ компонентов разрешающих переменных; $\vec{f}_{i}\left(x_{i}, \vec{y}_{i}, \vec{z}_{i}, \vec{\mu}_{i}, \vec{q}_{i}\right)$ - вектор-функция $n$ компонентов правых частей системы дифференциальных уравнений; $\vec{z}_{i}-$ вектор дополнительных переменных, т.е. 
переменных, не входящих под знак производной в системе (1), а рассчитываемых из дополнительных алгебраических соотношений $(2) ; \vec{\varphi}_{i}\left(x_{i}, \vec{y}_{i}, \vec{z}_{i}, \vec{\mu}_{i}, \vec{q}_{i}\right)$ вектор-функция нелинейных дополнительных алгебраических соотношений; $\vec{q}_{i}(x, t)$ - вектор-функция из $l$ компонентов поверхностных нагрузок; $\vec{\mu}_{i}-$ вектор параметров задачи; $\vec{d}_{j}$ - вектор сосредоточенных нагрузок в точках сопряжения сегментов конструкции; $M_{i}$ - матрица инерционных свойств элемента конструкции; $N$ - число сегментов краевой задачи.

При построении алгоритма решения задачи (5)-(8) представим нагрузку, действующую на деформируемый элемент, суммой заданных поверхностных нагрузок и инерционных нагрузок:

$$
\vec{q}_{i}^{*}(x, t)=\vec{q}_{i}(x, t)+M_{i} \frac{\partial^{2} \vec{y}_{i}}{\partial t^{2}} .
$$

При использовании метода дифференцирования по параметру для решения системы (5)-(8) вводится параметр нагрузки $\alpha$, т.е. нагрузка (3) записывается в виде

$$
\vec{q}_{i}^{* *}(x, t)=\alpha \vec{q}_{i}^{*}(x, t) .
$$

Используя $m$-точечную аппроксимацию вектора ускорений для момента времени $t=t_{k}, k \geqslant m$, запишем

$$
\left.\frac{\partial^{2} \vec{y}}{\partial t^{2}}\right|_{t=t_{k}}=\sum_{j=1}^{m} \beta_{j} \vec{y}_{m+1-j}
$$

Тогда с учетом представления (4) система уравнений (1) для моментов времени $t_{1} \leqslant t_{k}<t_{m}$ принимает вид

$$
\begin{aligned}
& \frac{d \vec{y}_{i, 1}}{d x_{i}}=\vec{F}_{i, 1}\left(x_{i}, \vec{y}_{i, 1}, \vec{z}_{i, 1}, \vec{\mu}_{i, 1}, \vec{q}_{i, 1}, t_{1}, \alpha\right), \\
& \quad \vdots \\
& \frac{d \vec{y}_{i, m-1}}{d x_{i}}=\vec{F}_{i, m-1}\left(x_{i}, \vec{y}_{i, m-1}, \vec{y}_{i, m-2}, \ldots, \vec{y}_{i, 1}, \vec{z}_{i, m-1}, \vec{\mu}_{i, m-1}, \vec{q}_{i, m-1}, t_{m-1}, \alpha\right),
\end{aligned}
$$

а для моментов времени $t_{k} \geqslant t_{m}-$

$$
\frac{d \vec{y}_{i, k}}{d x_{i}}=\vec{F}_{i, k}\left(x_{i}, \vec{y}_{i, k}, \vec{y}_{i, k-1}, \vec{y}_{i, k-2}, \ldots, \vec{y}_{i, k-m+1}, \vec{z}_{i, k}, \vec{\mu}_{i, k}, \vec{q}_{i, k}, t_{k}, \alpha\right),
$$

где $i \in[1, N]$.

При этом на первых $(m-1)$ шагах по времени для аппроксимации вектора ускорений используются законтурные точки [25]. Таким образом, для $k$-того регулярного шага по времени полная система уравнений, описывающих динамическое поведение конструкции, имеет вид

$$
\begin{gathered}
\frac{d \vec{y}_{i, k}}{d x_{i}}=\vec{F}_{i, k}\left(x_{i}, \vec{y}_{i, k}, \vec{y}_{i, k-1}, \vec{y}_{i, k-2}, \ldots, \vec{y}_{i, k-m+1}, \vec{z}_{i, k}, \vec{\mu}_{i, k}, \vec{q}_{i, k}, t_{k}, \alpha\right), \\
\vec{\varphi}_{i, k}\left(x_{i}, \vec{y}_{i, k}, \vec{z}_{i, k}, \vec{\mu}_{i, k}, \vec{q}_{i, k}, \alpha\right)=\overrightarrow{0}, \quad i \in[1, N]
\end{gathered}
$$


с условиями сопряжения сегментов конструкции

$$
\vec{y}_{j, k}\left(x_{j, e}\right)=\vec{y}_{j+1, k}\left(x_{j+1, b}\right)+\vec{d}_{j+1, k}, \quad j \in[1, N-1]
$$

и граничными условиями

$$
\vec{\psi}_{1, k}\left(x_{1}, \vec{y}_{1, k}, \vec{q}_{k}, \vec{\mu}_{k}, \tau_{k}\right)=\overrightarrow{0}, \quad 1 \rightleftharpoons 2 .
$$

Тогда при дифференцировании по некоторому заранее выбранному параметру $T$ системы уравнений (5) получим

$$
\frac{d \dot{\vec{y}}_{i, k}}{d x_{i}}=\frac{\partial \vec{F}_{i, k}}{\partial \vec{y}_{i, k}} \dot{\vec{y}}_{i, k}+\frac{\partial \vec{F}_{i, k}}{\partial \vec{z}_{i, k}} \dot{\vec{z}}_{i, k}+\frac{\partial \vec{F}_{i, k}}{\partial \vec{q}_{i, k}^{* *}} \frac{\partial \vec{q}_{i, k}^{* *}}{\partial T}
$$

где

$$
\frac{\partial{\overrightarrow{q_{i}}}_{i, k}^{* *}}{\partial T}=\dot{\alpha}\left(\vec{q}_{i, k}+\sum_{j=1}^{m} \beta_{j} \vec{y}_{k+1-j}\right)+\alpha\left(\dot{\vec{q}}_{i, k}+\beta_{1} \dot{\vec{y}}_{k}\right) .
$$

Точка здесь обозначает дифференцирование по параметру $T$.

Отметим, что решение задачи проводится последовательно на каждом временном слое, поэтому при дифференцировании по параметру аппроксимированного вектора ускорений скорости векторов разрешающих переменных по параметру предыдущих шагов по времени полагаются равными нулю.

Таким образом, при дифференцировании по параметру $T$ системы (5)(8), выражая из продифференцированного соотношения (6) скорости дополнительных переменных по параметру $\dot{\vec{z}}_{i, k}$, получим квазилинейную краевую задачу, которую можно представить в виде

$$
\begin{aligned}
\frac{d \dot{\overrightarrow{y_{i, k}}}}{d x_{i}}=A_{i, k}\left(x_{i}, \vec{y}_{i, k}, \vec{z}_{i, k},\right. & \left.\vec{q}_{i, k}, \vec{\mu}_{i, k}, \alpha\right) \dot{\vec{y}}_{i, k}+ \\
& +\vec{b}_{i, k}\left(x_{i}, \vec{y}_{i, k}, \vec{z}_{i, k}, \vec{q}_{i, k}, \vec{\mu}_{i, k}, \vec{y}_{i, k-1}, \ldots, \vec{y}_{i, k-m+1}\right) \dot{\alpha}, \\
\dot{\vec{y}}_{j, k}\left(x_{j, e}\right)= & \dot{\vec{y}}_{j+1, k}\left(x_{j+1, b}\right)+\dot{\vec{d}}_{j+1, k}, \quad j \in[1, N-1],
\end{aligned}
$$

$$
\begin{aligned}
B_{1, k}\left(x_{1}, \vec{y}_{1, k}, \vec{z}_{1, k}, \vec{\mu}_{1, k}, \vec{q}_{1, k}, \alpha\right) \dot{\vec{y}}_{1}+ & \\
& +\vec{b}_{1, k}\left(x_{1}, \vec{y}_{1, k}, \vec{z}_{1, k}, \vec{\mu}_{1, k}, \vec{q}_{1, k}\right) \dot{\alpha}=\overrightarrow{0}, \quad 1 \rightleftharpoons 2 .
\end{aligned}
$$

Как и обычно, при использовании метода дифференцирования по параметру квазилинейная краевая задача дополняется задачей Коши относительно искомых векторов разрешающих и дополнительных переменных и параметра нагрузки $\alpha$ :

$$
\frac{d \vec{y}_{i, j}}{d T}=\dot{\vec{y}}_{i, j}\left(\vec{y}_{i, j}, x_{i, j}, T\right), \quad \frac{d \vec{z}_{i, j}}{d T}=\dot{\vec{z}}_{i, j}\left(\vec{y}_{i, j}, x_{i, j}, T\right), \quad \frac{d \alpha}{d T}=\dot{\alpha}\left(\vec{y}_{i, j}, T\right) .
$$

Здесь $j \in\left[1, M_{i}\right]$, где $M_{i}$ - число точек дискретизации $i$-того сегмента конструкции, а индекс момента времени $k$ опущен. 
Алгоритм решения взаимосвязанных задач (9)-(11) и (12) и его особенности, связанные с реализацией применительно к задачам статики мягких оболочек, представлены в [26]. Для задач динамики поиск решения проводится при значениях параметра $\alpha \in[0,1]$, где решение при $\alpha=1$ соответствует решению исходной задачи (5)-(8) с точностью до принятой аппроксимации вектора ускорений.

\section{2. Динамическое раздувание шарнирно опертой полусферы из} неогуковского материала (пример). Систему уравнений для описания динамического поведения мягкой оболочки получим с использованием принципа наименьшего действия Остроградского-Гамильтона. Она включает в себя следующие соотношения:

- уравнения движения:

$$
\begin{gathered}
\frac{\partial T_{1 x}}{\partial \alpha}=\frac{A}{R_{1}} T_{1 z}+\frac{1}{A} \frac{d B}{d \alpha} T_{2 y}-A B p \vartheta_{1}\left(1+e_{2}\right)+A B \rho h \frac{\partial^{2} u}{\partial t^{2}}, \\
\frac{\partial T_{1 z}}{\partial \alpha}=-\frac{A}{R_{1}} T_{1 x}-\frac{B}{R_{2}} T_{2 y}+A B p\left(1+\varepsilon_{1}\right)\left(1+e_{2}\right)-A B \rho h \frac{\partial^{2} w}{\partial t^{2}} ;
\end{gathered}
$$

- геометрические дифференциальные уравнения:

$$
\frac{\partial u}{\partial S}=A \varepsilon_{1}-\frac{A}{R_{1}} w, \quad \frac{\partial w}{\partial S}=-A \vartheta_{1}+\frac{A}{R_{1}} u ;
$$

- дополнительные алгебраические соотношения:

$$
\begin{gathered}
e_{2}=\frac{1}{A B} \frac{d B}{d \alpha} u+\frac{1}{R_{2}} w, \quad e_{1}+\frac{1}{2} e_{1}^{2}=\varepsilon_{1}+\frac{1}{2}\left(\varepsilon_{1}^{2}+\vartheta_{1}^{2}\right) ; \\
T_{1 x}=T_{1}^{*}\left(1+\varepsilon_{1}\right) B, \quad T_{2 y}=T_{2}^{*}\left(1+e_{2}\right) A, \quad T_{1 z}=T_{1}^{*} \vartheta_{1} B ; \\
T_{1}^{*}=T_{1} \frac{1+e_{2}}{1+e_{1}}, \quad T_{2}^{*}=T_{2} \frac{1+e_{1}}{1+e_{2}}
\end{gathered}
$$

- и в общем случае нелинейные физические соотношения:

$$
T_{1}=T_{1}\left(e_{1}, e_{2}\right), \quad T_{2}=T_{2}\left(e_{1}, e_{2}\right) .
$$

Все обозначения соответствуют принятым в [30].

Рассмотрим раздувание шарнирно опертой полусферы внезапно приложенным постоянным во времени равномерно распределенным по меридиану давлением. Для определенности примем, что материал оболочки является неогуковским, для которого функция упругого потенциала имеет вид [15]:

$$
W=C\left(\lambda_{1}^{2}+\lambda_{2}^{2}+\lambda_{3}^{2}-3\right) .
$$

Здесь $\lambda_{i}=1+e_{i}, i=1,2,3$, а $C$ - параметр материала оболочки.

Используя связь функции упругого потенциала с напряжениями в несжимаемом изотропном материале [31], можно получить следующие физические соотношения:

$$
T_{1}=2 C h_{0}\left(\frac{\lambda_{1}}{\lambda_{2}}-\frac{1}{\left(\lambda_{1} \lambda_{2}\right)^{3}}\right), \quad 1 \rightleftharpoons 2,
$$

где $h_{0}$ - толщина оболочки в начальном состоянии. 
Для аппроксимации ускорения применим четырехточечную схему метода Хуболта [28] и трехточечную схему центральных разностей. Кроме того, при реализации предлагаемого алгоритма необходимо учесть особенности расчета мягких оболочек, указанные в [26]. В частности, необходимо задать малое предварительное давление, действующее на оболочку в начальный момент времени, и выполнить регуляризацию системы уравнений движения оболочки. Численные эксперименты показали, что при решении задачи динамики достаточно провести регуляризацию лишь на первом шаге по времени. Параметр продолжения решения был выбран в форме, предложенной В. И. Шалашилиным [32].

Необходимо также отметить, что в связи с плохой обусловленностью рассматриваемой задачи построение ее решения невозможно без использования метода сегментации. Указанный метод переводит двухточечную краевую задачу, формируемую из системы уравнений динамики мягкой оболочки при применении описанного выше метода прямых, к многоточечной. Таким образом, несмотря на то, что меридиан оболочки представляет собой однородную кривую, для построения численного решения необходимо представление этой кривой совокупностью сегментов с условиями сопряжения, характеризующими неразрывность меридиана.

На рис. 1 показана зависимость прогиба полюса полусферы, раздуваемой равномерно распределенной по меридиану нагрузкой $p^{*}=0.02$, отнесенного к ее начальному радиусу, от времени для значений шагов по времени $d \tau=0.5$, $d \tau=0.25, d \tau=0.125, d \tau=0.08$ при четырехточечной аппроксимации ускорения; на рис. 2 - аналогичные результаты при трехточечной аппроксимации. Отношение радиуса оболочки к ее толщине в начальном состоянии принято равным $R_{0} / h_{0}=100$. Безразмерные величины времени и нагрузки связаны с размерными следующими соотношениями:

$$
p^{*}=\frac{p}{C}, \quad \tau=t \sqrt{\frac{C}{R_{0}^{2} \rho}},
$$

где $\rho$ - плотность материала оболочки.

В таблице указаны значения периода колебаний оболочки, полученные в результате расчетов. При этом точное значение безразмерного периода колебаний оболочки для данной задачи составляет $T=2.31$, а величины амплитуды прогиба, отнесенного к начальному радиусу оболочки, $-w_{0}=0.38$ [15].

Как следует из представленных графиков и таблицы, на результаты расчета влияет выбор как шага по времени, так и схемы аппроксимации ускорения. Рис. 1 и 2 демонстрируют особенности численного решения задач динамики, проявляющиеся при использовании конечно-разностной аппроксимации ускорения и отмеченные в работах [25, 29], т.е. затухание амплитуды колебаний при использовании слишком большого шага по времени и уменьшение периода колебаний при уменьшении шага, причем затухание является наиболее выраженным для случая трехточечной аппроксимации. При этом с увеличением шага по времени решение затухает к решению статической задачи при приложении постоянной нагрузки рассматриваемой величины. Такие результаты представляются обоснованными, т.к. в предлагаемом алгоритме инерционные слагаемые в разрешающей системе уравнений рассматриваются как внешняя нагрузка, действующая на оболочку, и, следовательно, 
Рис. 1. Зависимость прогиба полюса раздуваемой постоянным давлением полусферы от времени при четырехточечной аппроксимации ускорения: $1-d \tau=0.5 ; 2-$ $d \tau=0.25 ; 3-d \tau=0.125 ; 4-d \tau=0.08$

[Figure 1. Time dependence of inflated by constant pressure hemisphere pole at four-point acceleration approximation: $1-$ $d \tau=0.5 ; 2-d \tau=0.25 ; 3-d \tau=0.125 ;$

$$
4-d \tau=0.08
$$

Рис. 2. Зависимость прогиба полюса раздуваемой постоянным давлением полусферы от времени при трехточечной аппроксимации ускорения: $1-d \tau=0.5 ; 2-d \tau=0.25$;

$$
3-d \tau=0.125 ; 4-d \tau=0.08
$$

[Figure 2. Time dependence of inflated by constant pressure hemisphere pole at three-point acceleration approximation: $1-$ $d \tau=0.5 ; 2-d \tau=0.25 ; 3-d \tau=0.125 ;$

$$
4-d \tau=0.08
$$
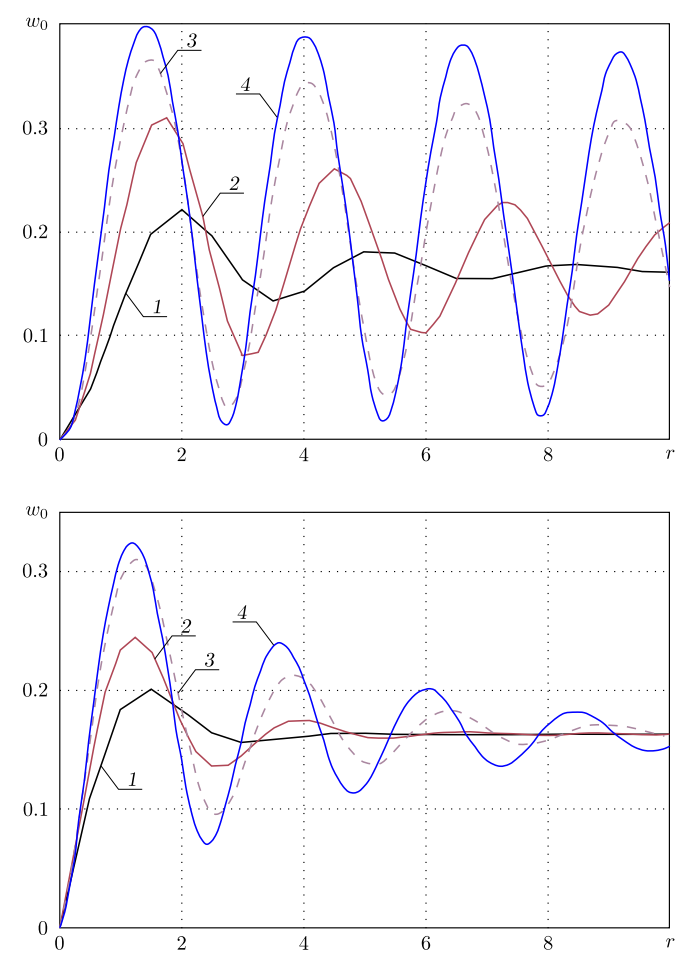

Значения периода колебаний полусферы при различных шагах по времени [The values of hemisphere vibrations period for different values of time step]

\begin{tabular}{l||c|c}
\hline \multicolumn{1}{c||}{$d \tau$} & \multicolumn{2}{|}{$\begin{array}{c}\text { Значения } T \text { для аппроксимации ускорения } \\
\text { [Values of } T \text { for acceleration approximation] }\end{array}$} \\
\cline { 2 - 3 } & $\begin{array}{c}\text { для четырехточечной } \\
\text { [for four-point] }\end{array}$ & $\begin{array}{c}\text { для трехточечной } \\
\text { [for three-point] }\end{array}$ \\
\hline 0.5 & 3 & - \\
0.25 & 2.75 & 2.75 \\
0.125 & 2.625 & 2.5 \\
0.08 & 2.56 & 2.4 \\
\hline
\end{tabular}

вносят вклад в значение амплитуды колебаний. Чем больше выбранная величина шага по времени, тем большая погрешность вносится в аппроксимацию ускорения в сторону уменьшения его величины и, следовательно, тем с большей погрешностью определяется амплитуда также в сторону уменьшения. Решение задачи проводится последовательно на каждом шаге по времени, и, таким образом, с каждым шагом происходит накопление погрешностей расчета, обусловленных аппроксимацией ускорения. Очевидно, что чем больше величина шага по времени, тем быстрее произойдет уменьшение амплитуд колебаний и, как следствие, аппроксимированного значения ускорения вплоть до нуля, что соответствует системе уравнений статического деформирования оболочки.

При этом, как показала практика вычислений, существует некоторое минимально допустимое для расчетов значение шага по времени, определяе- 
мое в результате численного эксперимента. Так, для рассматриваемой задачи наиболее близкие к теоретически определенным значения указанных величин получены в результате расчета по четырехточечной схеме аппроксимации ускорения при величине шага по времени $d \tau=0.08$ и составляют $T=2.56$, $w_{0}=0.4$ соответственно (амплитуда прогиба определяется на первом периоде колебаний, когда ее затухание, обусловленное особенностями вычислительного алгоритма, минимально). Отметим, что даже при этом значении шага не удается получить незатухающее решение. Однако провести расчеты при значении шага $d \tau<0.08$ оказалось невозможным вследствие либо потери устойчивости счета, либо отсутствия сходимости итераций. По-видимому, отмеченные особенности являются следствием ухудшения обусловленности матрицы Якоби разрешающей системы уравнений при уменьшении шага по времени. Таким образом, ввиду вычислительной сложности рассматриваемой задачи возможность получения незатухающего решения ограничена ее обусловленностью, а использование предлагаемого алгоритма требует обоснованного выбора шага интегрирования по времени.

Необходимо также подчеркнуть, что результаты аналитического решения задачи о раздувании сферы могут служить лишь для качественной оценки точности численного решения, но их нельзя считать точным решением рассматриваемой в примере задачи, т.к. аналитическое решение получено в предположении отсутствия предварительного напряженного состояния оболочки, а реализация численного алгоритма требует регуляризации решения, т.е., в том числе, задания некоторой величины предварительного давления на первом шаге по времени [26].

Отметим, что предлагаемый алгоритм впервые позволяет определить как временное, так и пространственное распределение компонент напряженнодеформированного состояния оболочки. Для рассматриваемой задачи последнее является простейшим, т.е. зависимость компонент от меридиональной координаты отсутствует, но выбор такой задачи обусловлен лишь необходимостью тестирования алгоритма и сравнения с имеющимися в литературе результатами аналитического решения.

Подчеркнем, что в проведенных расчетах, по-видимому, впервые при численном решении задачи динамического раздувания мягкой оболочки получены значения прогиба оболочки порядка половины ее радиуса. При этом, в отличие от работ большинства исследователей динамики мягкооболочечных конструкций, рассмотренная в качестве примера задача является лишь одной из широкого класса задач, охватываемых предлагаемым алгоритмом, а функция упругого потенциала материала оболочки может быть произвольной.

Заключение. Предложенный в работе подход к решению нелинейных задач динамики мягкооболочечных конструкций отличается от способов решения, разработанных другими авторами, возможностью численного исследования действительно больших перемещений и деформаций с учетом как геометрической, так и физической нелинейности. При этом предлагаемый алгоритм ориентирован на анализ целого класса мягкооболочечных конструкций, динамическое поведение которых может быть описано системой нелинейных уравнений в частных производных с дополнительными нелинейными алгебраическими соотношениями. Наиболее существенным ограничением 
является лишь предположение о зависимости компонент вектора разрешающих переменных только от одной обобщенной координаты и неразветвленности конструкции. При этом практика расчетов показала, что чем меньше ограничений накладывается на исходные данные решаемой задачи (геометрия конструкции, свойства материала, условия нагружения и закрепления, диапазон исследуемых перемещений и деформаций), тем более тщательным должен быть подбор параметров вычислительного алгоритма решения такой задачи. Это связано с тем, что при получении численного решения в каждом конкретном случае условий работы конструкции могут возникать различные вычислительные сложности. Этим и обусловлены описанные в работе ограничения, связанные с выбором шага по времени, заданием предварительного напряженного состояния, необходимостью использования метода сегментации и т.п., причем характер ограничений можно установить только в процессе проведения расчетов. Однако достоинством такого подхода к решению задач динамики мягких оболочек, в отличие от численных алгоритмов, предложенных другими авторами, является возможность исследования поведения конструкции в диапазоне перемещений, величина которых сопоставима с геометрическими размерами конструкции, а по сравнению с работами, в которых решения подобных задач получены аналитически, здесь имеется возможность анализа распределения компонент напряженно-деформированного состояния конструкции не только во времени, но и по координате.

Конкурирующие интересы. Я заявляю об отсутствии явных и потенциальных конфликтов интересов, связанных с публикацией настоящей статьи.

Авторская ответственность. Я несу полную ответственность за предоставление окончательной версии рукописи в печать. Окончательная версия рукописи мною одобрена.

Финансирование. Исследование выполнялось без финансирования.

\section{Библиографический список}

1. Друзь Б. И., Друзь И. Б. Теория мягких оболочек. Владивосток: Морской гос. ун-т, 2003. 381 c.

2. Ридель В. В., Гулин Б. В. Динамика мягких оболочек. М.: Наука, 1990. 204 с.

3. Гимадиев Р. Ш. Динамика мягких оболочек парашютного типа. Казань: Казан. гос. энерг. ун-т, 2006. 208 с.

4. Libai A., Simmonds J. G. The Nonlinear Theory of Elastic Shells. Cambridge: Cambridge Univ. Press, 1998. 560 pp. https://doi.org/10.1017/CB09780511574511.

5. Лялин В. В., Морозов В. И., Пономарев А. Т. Парашютные системы. Проблемы и методы их решения. М.: Физматлит, 2009. 576 с.

6. Knowles J. K. On a class of oscillations in the finite deformation theory of elasticity // J. Appl. Mech., 1962. vol. 29, no. 2. pp. 283-286. https://doi.org/10.1115/1.3640542.

7. Akkas N. On the dynamic snap-out instability of inflated non-linear spherical membranes // Int. J. Non-Linear Mechanics, 1978. vol. 13, no. 3. pp. 177-183. https://doi.org/10.1016/ 0020-7462 (78) 90006-9.

8. Calderer C. The dynamical behaviour of nonlinear elastic spherical shells // J. Elasticity, 1983. vol. 13. pp. 17-47. https://doi.org/10.1007/bf00041312.

9. Verron E., Khayat R. E., Derdouri A., Peseux B. Dynamic inflation of hyperelastic spherical membranes // J. Rheology, 1999. vol. 43, no. 5. pp. 1083-1097. https://doi.org/10.1122/ 1.551017. 
10. Yuan X. G., Zhang R. J., Zhang H. W. Controllability conditions of finite oscillations of hyperelastic cylindrical tubes composed of a class of Ogden material models // Comput. Mater. Continua, 2008. vol. 7, no. 3. pp. 155-166.

11. Ren J. Dynamical response of hyper-elastic cylindrical shells under periodic load// Appl. Math. Mech., 2008. vol.29, no.10. pp. 1319-1327. https://doi.org/10.1007/ s10483-008-1007-x.

12. Ren J. Dynamics and destruction of internally pressurized incompressible hyper-elastic spherical shells // Int. J. Eng. Sci., 2009. vol.47, no.7-8. pp. 745-753. https://doi.org/ 10.1016/j.ijengsci.2009.02.001.

13. Yong H., He X., Zhou Y. Dynamics of a thick-walled dielectric elastomer spherical shell // Int. J. Eng. Sci., 2011. vol.49, no. 8. pp. 792-800. https://doi.org/10.1016/j.ijengsci. 2011.03 .006$.

14. Ju Y., Niu D. On a class of differential equations of motion of hyperelastic spherical membranes // Appl. Math. Sci., 2012. vol. 6, no. 83. pp. 4133-4136.

15. Rodríguez-Martínez J. A., Fernández-Sáez J., Zaera R. The role of constitutive relation in the stability of hyper-elastic spherical membranes subjected to dynamic inflation // Int. J. Eng. Sci., 2015. vol. 93. pp. 31-45. https://doi.org/10.1016/j.ijengsci.2015.04.004.

16. Zhao Zh., Zhang W., Zhang H., Yuan X. Some interesting nonlinear dynamic behaviors of hyperelastic spherical membranes subjected to dynamic loads // Acta Mechanica, 2019. vol. 230, no. 8. pp. 3003-3018. https://doi.org/10.1007/s00707-019-02467-y.

17. Shahinpoor M., Balakrishnan R. Large amplitude oscillations of thick hyperelastic cylindrical shells // Int. J. Non-Linear Mechanics, 1978. vol. 13, no. 5-6. pp. 295-301. https:// doi .org/10.1016/0020-7462(78)90035-5.

18. Wang A. S. D., Ertepinar A. Stability and vibrations of elastic thick-walled cylindrical and spherical shells subjected to pressure// Int. J. Non-Linear Mechanics, 1972. vol. 7, no. 5. pp. 539-555. https://doi.org/10.1016/0020-7462(72)90043-1.

19. Akyüz U., Ertepinar A. Stability and asymmetric vibrations of pressurized compressible hyperelastic cylindrical shells // Int. J. Non-Linear Mechanics, 1999. vol. 34, no. 3. pp. 391404. https://doi.org/10.1016/s0020-7462(98)00015-8.

20. Zhu Y., Luo X. Y., Wang H. M., Ogden R. W., Berry C. Three-dimensional non-linear buckling of thick-walled elastic tubes under pressure// Int. J. Non-Linear Mechanics, 2013. vol. 48, no. 1. pp. 1-14. https://doi.org/10.1016/j.ijnonlinmec.2012.06.013.

21. Soares R. M., Gonçalves P. B. Large-amplitude nonlinear vibrations of a Mooney-Rivlin rectangular membrane // J. Sound Vibration, 2014. vol. 333, no. 13. pp. 2920-2935. https:// doi.org/10.1016/j.jsv.2014.02.007.

22. Gorissen B., Melancon D., Vasios N., Torbati M., Bertoldi K. Inflatable soft jumper inspired by shell snapping// Science Robotics, 2020. vol.5, no. 42, eabb1967. https://doi.org/10. 1126/scirobotics.abb1967.

23. Rogers C., Saccomandi G., Vergori L. Helmholtz-type solitary wave solutions in nonlinear elastodynamics // Ricerche Mat., 2019. vol.69, no.1. pp. 327-341. https://doi.org/10. 1007/s11587-019-00464-w.

24. Pucci E., Saccomandi G., Vergori L. Linearly polarized waves of finite amplitude in prestrained elastic materials // Proc. R. Soc. Lond., Ser. A, 2019. vol.475, no. 2226. https:// doi.org/10.1098/rspa.2018.0891.

25. Johnson D. E., Greif R. Dynamic response of a cylindrical shell. Two numerical methods // AIAA Journal, 1965. vol. 4, no.3. pp. 486-494. https://doi.org/10.2514/3.3462.

26. Коровайцева Е. А. О некоторых особенностях решения задач статики мягких оболочек вращения при больших деформациях // Трудъ МАИ, 2020. Т.114. 34 pp. https://doi . org/10.34759/trd-2020-114-04.

27. Шаповалов Л. А. Уравнения эластики тонкой оболочки при неосесимметричной деформации // Изв. АН СССР. Мех. тв. тела, 1976. № 3. С. 62-72.

28. Houbolt J. C. A recurrence matrix solution for the dynamic response of elastic aircraft // J. Aeronaut. Sci., 1950. vol.17, no. 9. pp. 540-550. https://doi.org/10.2514/8.1722. 
29. Levy S., Kroll W. D. Errors introduced by finite space and time increments in dynamic response computation// J. Res. Natl. Bur. Stand., 1953. vol.51, no.1. pp. 57-68. https:// doi.org/10.6028/jres.051.006.

30. Коровайцева Е. А. Смешанные уравнения теории мягких оболочек // Труды МАИ, 2019. T. 108. 17 pp. https://doi.org/10.34759/trd-2019-108-1.

31. Amabili M. Nonlinear Mechanics of Shells and Plates in Composite, Soft and Biological Materials. Cambridge: Cambridge Univ. Press, 2018. xvi+568 pp. https://doi.org/10. 1017/9781316422892.

32. Григолюк Э. И., Шалашилин В. И. Метод продолжения по параметру в нелинейных задачах механики твердого деформируемого тела. М.: Наука, 1988. 232 с. 


\title{
MSC: 74B20, 74K25, 74H15
}

\section{Parameter differentiation method in solution of axisymmetric soft shells stationary dynamics nonlinear problems}

\section{(C) E. A. Korovaytseva}

Lomonosov Moscow State University, Institute of Mechanics,

1, Michurinsky prospekt, Moscow, 119192, Russian Federation.

\begin{abstract}
An algorithm of axisymmetric unbranched soft shells nonlinear dynamic behaviour problems solution is suggested. The algorithm does not impose any restrictions on deformations or displacements range, material properties, conditions of fixing or meridian form of the structure. Mathematical statement of the problem is given in vector-matrix form and includes system of partial differential equations, system of additional algebraic equations, structure segments coupling conditions, initial and boundary conditions. Partial differential equations of motion are reduced to nonlinear ordinary differential equations using method of lines. Obtained equation system is differentiated by calendar parameter. As a result problem solution is reduced to solving two interconnected problems: quasilinear multipoint boundary problem and nonlinear Cauchy problem with right-hand side of a special form. Features of represented algorithm using in application to the problems of soft shells dynamics are revealed at its program realization and are described in the study. Three- and four-point finite difference schemes are used for acceleration approximation. Algorithm testing is carried out for the example of hinged hemisphere of neo-hookean material dynamic inflation. Influence of time step and acceleration approximation scheme choice on solution results is investigated.
\end{abstract}

Keywords: soft shell, hyperelastic material, dynamic inflation, method of lines, parameter differentiation method, physical nonlinearity, geometrical nonlinearity.

Received: 23 ${ }^{\text {rd }}$ March, $2021 /$ Revised: $17^{\text {th }}$ May, $2021 /$

Accepted: $25^{\text {th }}$ August, $2021 /$ First online: $30^{\text {th }}$ September, 2021

\section{Research Article}

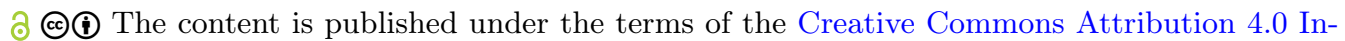
ternational License (http://creativecommons.org/licenses/by/4.0/)

Please cite this article in press as:

Korovaytseva E. A. Parameter differentiation method in solution of axisymmetric soft shells stationary dynamics nonlinear problems, Vestn. Samar. Gos. Tekhn. Univ., Ser. Fiz.-Mat. Nauki [J. Samara State Tech. Univ., Ser. Phys. Math. Sci.], 2021, vol. 25, no. 3, pp. 556-570. https://doi.org/10.14498/vsgtu1855 (In Russian).

Author's Details:

Ekaterina A. Korovaytseva (10) https://orcid.org/0000-0001-6663-8689

Cand. Tech. Sci.; Senior Researcher; Lab. of Dynamic Tests; e-mail: katrell@mail.ru 
Competing interests. I declare that I have no apparent or potential conflicts of interest related to the publication of this article.

Authors' contributions and responsibilities. I take full responsibility for submit the final manuscript to print. I approved the final version of the manuscript.

Funding. This research received no specific grant from any funding agency in the public, commercial, or not-for-profit sectors.

\section{References}

1. Druz' B. I., Druz' I. B. Teoriia miagkikh obolochek [Theory of Soft Shells]. Vladivostok, Maritime State Univ., 2003, 381 pp. (In Russian)

2. Ridel' V. V., Gulin B. V. Dinamika miagkikh obolochek [Dynamics of Soft Shells]. Moscow, Nauka, 1990, 204 pp. (In Russian)

3. Gimadiev R. Sh. Dinamika miagkikh obolochek parashiutnogo tipa [Dynamics of Soft Parachute-Type Shells]. Kazan, Kazan State Energ. Univ., 2006, 208 pp. (In Russian)

4. Libai A., Simmonds J. G. The Nonlinear Theory of Elastic Shells. Cambridge, Cambridge Univ. Press, 1998, 560 pp. https://doi.org/10.1017/CB09780511574511.

5. Lyalin V. V., Morozov V. I., Ponomarev A. T. Parashiutnye sistemy. Problemy i metody ikh resheniia [Parachute Systems. Problems and Methods of Their Solution]. Moscow, Fizmatlit, 2009, 576 pp. (In Russian)

6. Knowles J. K. On a class of oscillations in the finite deformation theory of elasticity, J. Appl. Mech., 1962, vol. 29, no.2, pp. 283-286. https://doi.org/10.1115/1.3640542.

7. Akkas N. On the dynamic snap-out instability of inflated non-linear spherical membranes, Int. J. Non-Linear Mechanics, 1978, vol. 13, no. 3, pp. 177-183. https://doi.org/10.1016/ 0020-7462 (78) 90006-9.

8. Calderer C. The dynamical behaviour of nonlinear elastic spherical shells, J. Elasticity, 1983, vol. 13, pp. 17-47. https://doi.org/10.1007/bf00041312.

9. Verron E., Khayat R. E., Derdouri A., Peseux B. Dynamic inflation of hyperelastic spherical membranes, J. Rheology, 1999, vol. 43, no. 5, pp. 1083-1097. https://doi.org/10.1122/1. 551017.

10. Yuan X. G., Zhang R. J., Zhang H. W. Controllability conditions of finite oscillations of hyperelastic cylindrical tubes composed of a class of Ogden material models, Comput. Mater. Continua, 2008, vol. 7, no. 3, pp. 155-166.

11. Ren J. Dynamical response of hyper-elastic cylindrical shells under periodic load, Appl. Math. Mech., 2008, vol.29, no.10, pp. 1319-1327. https://doi.org/10.1007/ s10483-008-1007-x.

12. Ren J. Dynamics and destruction of internally pressurized incompressible hyper-elastic spherical shells, Int. J. Eng. Sci., 2009, vol.47, no.7-8, pp. 745-753. https://doi.org/ 10.1016/j.ijengsci.2009.02.001.

13. Yong H., He X., Zhou Y. Dynamics of a thick-walled dielectric elastomer spherical shell, Int. J. Eng. Sci., 2011, vol.49, no. 8, pp. 792-800. https://doi.org/10.1016/j.ijengsci. 2011.03 .006$.

14. Ju Y., Niu D. On a class of differential equations of motion of hyperelastic spherical membranes, Appl. Math. Sci., 2012, vol. 6, no. 83, pp. 4133-4136.

15. Rodríguez-Martínez J. A., Fernández-Sáez J., Zaera R. The role of constitutive relation in the stability of hyper-elastic spherical membranes subjected to dynamic inflation, Int. J. Eng. Sci., 2015, vol. 93, pp. 31-45. https://doi.org/10.1016/j.ijengsci.2015.04.004.

16. Zhao Zh., Zhang W., Zhang H., Yuan X. Some interesting nonlinear dynamic behaviors of hyperelastic spherical membranes subjected to dynamic loads, Acta Mechanica, 2019, vol. 230, no. 8, pp. 3003-3018. https://doi.org/10.1007/s00707-019-02467-y.

17. Shahinpoor M., Balakrishnan R. Large amplitude oscillations of thick hyperelastic cylindrical shells, Int. J. Non-Linear Mechanics, 1978, vol.13, no. 5-6, pp. 295-301. https://doi. org/10.1016/0020-7462(78)90035-5. 
18. Wang A. S. D., Ertepinar A. Stability and vibrations of elastic thick-walled cylindrical and spherical shells subjected to pressure, Int. J. Non-Linear Mechanics, 1972, vol. 7, no. 5, pp. 539-555. https://doi.org/10.1016/0020-7462(72)90043-1.

19. Akyüz U., Ertepinar A. Stability and asymmetric vibrations of pressurized compressible hyperelastic cylindrical shells, Int. J. Non-Linear Mechanics, 1999, vol.34, no. 3, pp. 391404. https://doi.org/10.1016/s0020-7462(98)00015-8.

20. Zhu Y., Luo X. Y., Wang H. M., Ogden R. W., Berry C. Three-dimensional non-linear buckling of thick-walled elastic tubes under pressure, Int. J. Non-Linear Mechanics, 2013, vol. 48, no. 1, pp. 1-14. https://doi.org/10.1016/j.ijnonlinmec.2012.06.013.

21. Soares R. M., Gonçalves P. B. Large-amplitude nonlinear vibrations of a Mooney-Rivlin rectangular membrane, J. Sound Vibration, 2014, vol. 333, no. 13, pp. 2920-2935. https:// doi.org/10.1016/j.jsv.2014.02.007.

22. Gorissen B., Melancon D., Vasios N., Torbati M., Bertoldi K. Inflatable soft jumper inspired by shell snapping, Science Robotics, 2020, vol.5, no. 42, eabb1967. https://doi.org/10. 1126/scirobotics.abb1967.

23. Rogers C., Saccomandi G., Vergori L. Helmholtz-type solitary wave solutions in nonlinear elastodynamics, Ricerche Mat., 2019, vol.69, no.1, pp. 327-341. https://doi.org/ 10.1007/s11587-019-00464-w.

24. Pucci E., Saccomandi G., Vergori L. Linearly polarized waves of finite amplitude in prestrained elastic materials, Proc. R. Soc. Lond., Ser. A, 2019, vol.475, no. 2226. https:// doi.org/10.1098/rspa.2018.0891.

25. Johnson D. E., Greif R. Dynamic response of a cylindrical shell. Two numerical methods, AIAA Journal, 1965, vol. 4, no. 3, pp. 486-494. https://doi.org/10.2514/3.3462.

26. Korovaytseva E. A. On some features of soft shells of revolution static problems solution at large deformations, Trudy MAI, 2020, vol. 114, 34 pp. (In Russian). https://doi.org/ 10.34759/trd-2020-114-04

27. Shapovalov L. A. Elasticity equations for a thin shell at nonaxisymmetric deformation, Izv. Akad. Nauk SSSR. MTT, 1976, vol.3, pp. 62-72 (In Russian).

28. Houbolt J. C. A recurrence matrix solution for the dynamic response of elastic aircraft, J. Aeronaut. Sci., 1950, vol.17, no. 9, pp. 540-550. https://doi.org/10.2514/8.1722.

29. Levy S., Kroll W. D. Errors introduced by finite space and time increments in dynamic response computation, J. Res. Natl. Bur. Stand., 1953, vol.51, no.1, pp. 57-68. https:// doi.org/10.6028/jres.051.006.

30. Korovaytseva E. A. Combined equations of theory of soft shells, Trudy MAI, 2019, vol. 108, 17 pp. (In Russian). https://doi.org/10.34759/trd-2019-108-1

31. Amabili M. Nonlinear Mechanics of Shells and Plates in Composite, Soft and Biological Materials. Cambridge, Cambridge Univ. Press, 2018, xvi+568 pp. https://doi.org/10. $1017 / 9781316422892$.

32. Grigolyuk E. I., Shalashilin V. I. Problems of Nonlinear Deformation. The Continuation Method Applied to Nonlinear Problems in Solid Mechanics. Berlin, Springer, 1991, viii+262 pp. https://doi.org/10.1007/978-94-011-3776-8. 